ANNALES

POLONICI MATHEMATICI

$93.1(2008)$

\title{
Some further results on meromorphic functions that share two sets
}

\author{
by QI HAN and Hong-XUN YI (Jinan)
}

\begin{abstract}
This paper concerns the uniqueness of meromorphic functions and shows that there exists a set $\mathbf{S} \subset \mathbb{C}$ of eight elements such that any two nonconstant meromorphic functions $f$ and $g$ in the open complex plane $\mathbb{C}$ satisfying $E_{3}(\mathbf{S}, f)=E_{3)}(\mathbf{S}, g)$ and $\bar{E}(\infty, f)=\bar{E}(\infty, g)$ are identical, which improves a result of H. X. Yi. Also, some other related results are obtained, which generalize the results of G. Frank, E. Mues, M. Reinders, C. C. Yang, H. X. Yi, P. Li, M. L. Fang and H. Guo, and others.
\end{abstract}

1. Introduction and main results. In this paper, a meromorphic function will always mean meromorphic in the open complex plane $\mathbb{C}$. For any nonconstant meromorphic function $f$, we adopt the standard notations in Nevanlinna's value distribution theory of meromorphic functions such as the characteristic function $T(r, f)$, the proximity function $m(r, f)$ and the counting function $N(r, f)$ (reduced form $\bar{N}(r, f)$ ) of poles. Also, we denote by $S(r, f)$ any quantity satisfying $S(r, f)=o(T(r, f))$, possibly outside a set of finite linear measure in $\mathbb{R}$ that is not necessarily the same at each occurrence. We refer the reader to books [6] and [10] for more details on those notations.

Let $f$ be a nonconstant meromorphic function, let $a \in \mathbb{C}$ be a finite value, and let $k$ be a positive integer or infinity. We denote by $E(a, f)$ the set of zeros of $f-a$ (counting multiplicity), and by $\bar{E}(a, f)$ the set of zeros of $f-a$ (ignoring multiplicity). Also, we denote by $E_{k}(a, f)$ the set of zeros of $f-a$ with multiplicities less than or equal to $k$ (counting multiplicity). Obviously, $E(a, f)=E_{+\infty)}(a, f)$. If $a=\infty$, we define $E(\infty, f):=E(0,1 / f)$. $\bar{E}(\infty, f)$ and $E_{k)}(\infty, f)$ are similarly defined. For $a \in \mathbb{C} \cup\{\infty\}$, we denote by $N_{k)}(r, 1 /(f-a))$ the counting function corresponding to the set $E_{k)}(a, f)$, and by $N_{(k+1}(r, 1 /(f-a))$ the counting function corresponding to the set

2000 Mathematics Subject Classification: Primary 30D35; Secondary 30D20, 30D30.

Key words and phrases: entire function, meromorphic function, sharing sets, uniqueness.

Project supported by the NSFC (No. 10771121) and the RFDP (No. 20060422049). 
$E_{(k+1}(a, f):=E(a, f) / E_{k)}(a, f)$. Also, we denote by $\bar{N}_{k)}(r, 1 /(f-a))$ and $\bar{N}_{(k+1}(r, 1 /(f-a))$ the corresponding reduced forms of $N_{k)}(r, 1 /(f-a))$ and $N_{(k+1}(r, 1 /(f-a))$, respectively.

Let $\mathbf{S}$ be a subset in $\mathbb{C} \cup\{\infty\}$ of distinct elements, and let $k$ be a positive integer or infinity. Set

$$
E_{k)}(\mathbf{S}, f)=\bigcup_{a \in \mathbf{S}} E_{k)}(a, f) \quad \text { and } \quad \bar{E}(\mathbf{S}, f)=\bigcup_{a \in \mathbf{S}} \bar{E}(a, f) .
$$

Obviously, for $a \in \mathbb{C} \cup\{\infty\}, E_{k)}(\{a\}, f)=E_{k)}(a, f)$ and $\bar{E}(\{a\}, f)=$ $\bar{E}(a, f)$. For another nonconstant meromorphic function $g$, we say that $f$ and $g$ share the set $\mathbf{S} C M$ (respectively, $I M$ ) provided that $E(\mathbf{S}, f)=E(\mathbf{S}, g)$ (respectively, $\bar{E}(\mathbf{S}, f)=\bar{E}(\mathbf{S}, g)$ ). Evidently, if $\mathbf{S}$ contains only one element, then it coincides with the usual definition of CM (respectively, IM) shared values. On the other hand, the condition $E_{k)}(\mathbf{S}, f)=E_{k)}(\mathbf{S}, g)$ obviously implies $E_{j)}(\mathbf{S}, f)=E_{j)}(\mathbf{S}, g)$ for all $1 \leq j \leq k$.

It was F. Gross [3] who first considered the uniqueness of meromorphic functions that share sets of distinct elements instead of values. In 1976, he proposed the following two questions in [4].

Question I. Can one find two (or possibly even one) finite sets $\mathbf{S}_{j}$ $(j=1,2)$ such that any two nonconstant entire functions $f$ and $g$ satisfying $E\left(\mathbf{S}_{j}, f\right)=E\left(\mathbf{S}_{j}, g\right)(j=1,2)$ are identical?

QUESTION II. If the answer to Question I (for two finite sets) is positive, then how large have both sets to be?

Further, in 1982, F. Gross and C. C. Yang [5] showed that any two nonconstant entire functions $f$ and $g$ satisfying $E(\mathbf{S}, f)=E(\mathbf{S}, g)$ are identical, where $\mathbf{S}:=\left\{\omega \in \mathbb{C} \mid \omega+e^{\omega}=0\right\}$. Only about twenty years later, were Questions I and II completely answered by H. X. Yi who proved the following three theorems.

Theorem A (see [11]). Let $\mathbf{S}_{1}=\left\{\omega \mid \omega^{n}-1=0\right\}$ and $\mathbf{S}_{2}=\{a\}$, where $n$ is a positive integer such that $n \geq 5$, and $a$ is a constant such that $a \neq 0$ and $a^{2 n} \neq 1$. If $f$ and $g$ are two nonconstant entire functions satisfying $E\left(\mathbf{S}_{j}, f\right)=E\left(\mathbf{S}_{j}, g\right)$ for $j=1,2$, then $f \equiv g$.

Theorem B (see [12]). Let $\mathbf{S}=\left\{\omega \mid \omega^{n}+a \omega^{m}+b=0\right\}$, where $n$ and $m$ are positive integers such that $n \geq 15, n>m \geq 5$, and $n$ and $m$ are relatively prime, and $a$ and $b$ are nonzero constants such that the algebraic equation $\omega^{n}+a \omega^{m}+b=0$ has no multiple roots. If $f$ and $g$ are two nonconstant entire functions satisfying $E(\mathbf{S}, f)=E(\mathbf{S}, g)$, then $f \equiv g$.

Theorem C (see [15]). Let $\mathbf{S}_{1}=\left\{\omega \mid \omega^{3}+a \omega^{2}+b=0\right\}$ and $\mathbf{S}_{2}=\{0\}$, where $a$ and $b$ are nonzero constants such that the equation $\omega^{3}+a \omega^{2}+b$ 
$=0$ has no multiple roots. If $f$ and $g$ are two nonconstant entire functions satisfying $E\left(\mathbf{S}_{j}, f\right)=E\left(\mathbf{S}_{j}, g\right)$ for $j=1,2$, then $f \equiv g$. Obviously, $\min \left\{\imath\left(\mathbf{S}_{1}\right), \imath\left(\mathbf{S}_{2}\right)\right\} \geq 1$ and $\max \left\{\imath\left(\mathbf{S}_{1}\right), \imath\left(\mathbf{S}_{2}\right)\right\} \geq 3$, where $\imath(\mathbf{S})$ denotes the cardinality of $\mathbf{S}$.

Clearly, Theorems A and B answer Question I affirmatively, while Theorem C answers Question II completely, since examples are given in [15] to show the sharpness. See also [14] for some related results.

In 1998, G. Frank and M. Reinders obtained the following result which extends and improves Theorem B.

TheOREm D (see [2]). Let $n$ be a positive integer such that $n \geq 11$, and let $c$ be a constant such that $c \neq 0,1$. Then the polynomial

$$
P^{\mathrm{FR}}(\omega)=\frac{(n-1)(n-2)}{2} \omega^{n}-n(n-2) \omega^{n-1}+\frac{n(n-1)}{2} \omega^{n-2}-c
$$

has no multiple roots. Let $\mathbf{S}$ denote the zero set of $P^{\mathrm{FR}}(\omega)$. If $f$ and $g$ are two nonconstant meromorphic functions satisfying $E(\mathbf{S}, f)=E(\mathbf{S}, g)$, then $f \equiv g$.

In particular, if $f$ and $g$ are entire, then $n \geq 7$ suffices, which coincides with the main result of E. Mues and M. Reinders [9] on entire functions.

It is natural to consider the uniqueness of two nonconstant meromorphic functions that satisfy $E(\mathbf{S}, f)=E(\mathbf{S}, g)$ and $E(\infty, f)=E(\infty, g)$, a problem clearly inspired by Theorems B and D. The first result on this problem was obtained by P. Li and C. C. Yang:

Theorem E (see [7]). Let $\mathbf{S}=\left\{\omega \mid \omega^{n}+a \omega^{n-m}+b=0\right\}$, where $n$ and $m$ are two positive integers such that $m \geq 2, n>4 m+6$, and $n$ and $m$ are relatively prime, and $a$ and $b$ are nonzero constants such that the equation $\omega^{n}+a \omega^{n-m}+b=0$ has no multiple roots. If $f$ and $g$ are two nonconstant meromorphic functions satisfying $E(\mathbf{S}, f)=E(\mathbf{S}, g)$ and $E(\infty, f)=E(\infty, g)$, then $f \equiv g$.

From then on, many results on that problem have been obtained. In 1995, H. X. Yi [13], and independently P. Li and C. C. Yang [8], showed that there exists a set $\mathbf{S}$ of 11 elements such that any two nonconstant meromorphic functions $f$ and $g$ satisfying $E(\mathbf{S}, f)=E(\mathbf{S}, g)$ and $E(\infty, f)=E(\infty, g)$ are identical. In 1997, M. L. Fang and H. Guo in [1] exhibited a set $\mathbf{S}$ of nine elements with this property.

In 2002, H. X. Yi proved the following theorem.

TheOREM F (see [16]). Let $n$ be a positive integer such that $n \geq 8$, and let $a, b$ be nonzero constants such that $a b^{n-2} \neq 2$. Then the polynomial

$$
P(\omega)=a \omega^{n}-n(n-1) \omega^{2}+2 n(n-2) b \omega-(n-1)(n-2) b^{2}
$$


has no multiple roots. Let $\mathbf{S}$ denote the zero set of $P(\omega)$. If $f$ and $g$ are two nonconstant meromorphic functions satisfying $E(\mathbf{S}, f)=E(\mathbf{S}, g)$ and $\bar{E}(\infty, f)=\bar{E}(\infty, g)$, then $f \equiv g$.

In this paper, we prove the following five results, which are improvements on and supplements to the above theorems.

Theorem 1. Let $\mathbf{S}$ be the zero set of $P(\omega)$ given by (1.3) with $n \geq 7$. If $f$ and $g$ are two nonconstant entire functions satisfying $E_{2)}(\mathbf{S}, f)=E_{2)}(\mathbf{S}, g)$, then $f \equiv g$.

TheOREM 2. Let $\mathbf{S}$ be the zero set of $P(\omega)$ given by (1.3) with $n \geq 9$. If $f$ and $g$ are two nonconstant entire functions satisfying $E_{1)}(\mathbf{S}, f)=E_{1)}(\mathbf{S}, g)$, then $f \equiv g$.

TheOREM 3. Let $\mathbf{S}$ be the zero set of $P(\omega)$ given by (1.3) with $n \geq 8$. If $f$ and $g$ are two nonconstant meromorphic functions satisfying $E_{3}(\mathbf{S}, f)=$ $E_{3)}(\mathbf{S}, g)$ and $\bar{E}(\infty, f)=\bar{E}(\infty, g)$, then $f \equiv g$.

TheOREM 4. Let $\mathbf{S}$ be the zero set of $P(\omega)$ given by (1.3) with $n \geq 9$. If $f$ and $g$ are two nonconstant meromorphic functions satisfying $E_{2}(\mathbf{S}, f)=$ $E_{2)}(\mathbf{S}, g)$ and $\bar{E}(\infty, f)=\bar{E}(\infty, g)$, then $f \equiv g$.

THEOREM 5. Let $\mathbf{S}$ be the zero set of $P(\omega)$ given by (1.3) with $n \geq 12$. If $f$ and $g$ are two nonconstant meromorphic functions satisfying $E_{1)}(\mathbf{S}, f)=$ $E_{1)}(\mathbf{S}, g)$ and $\bar{E}(\infty, f)=\bar{E}(\infty, g)$, then $f \equiv g$.

REMARK 6 . Let $P(\omega)$ be given by (1.3), and let $n, a$ and $b$ be as in Theorem F. Set

$$
R(\omega)=\frac{a \omega^{n}}{n(n-1)(\omega-\alpha)(\omega-\beta)},
$$

where $\alpha$ and $\beta$ are the two distinct roots of the algebraic equation

$$
n(n-1) \omega^{2}-2 n(n-2) b \omega+(n-1)(n-2) b^{2}=0 .
$$

Then

$$
R^{\prime}(\omega)=\frac{a(n-2) \omega^{n-1}(\omega-b)^{2}}{n(n-1)(\omega-\alpha)^{2}(\omega-\beta)^{2}} .
$$

So $\omega=0$ is an $n$-fold root of $R(\omega)=0$, while $\omega=b$ is a triple root of $R(\omega)-a b^{n-2} / 2=0$. Since $a b^{n-2} \neq 0,2$, we have $R(\omega)-1=0$, and thus $P(\omega)=0$ has no multiple roots.

\section{Lemmas}

Lemma 1 (see [2]). Let

$$
Q(\omega)=(n-1)^{2}\left(\omega^{n}-1\right)\left(\omega^{n-2}-1\right)-n(n-2)\left(\omega^{n-1}-1\right)^{2} .
$$


Then

$$
Q(\omega)=(n-1)^{2}(\omega-1)^{4}\left(\omega-\gamma_{1}\right)\left(\omega-\gamma_{2}\right) \cdots\left(\omega-\gamma_{2 n-6}\right),
$$

where $\gamma_{j} \in \mathbb{C} \backslash\{0,1\}$ are pairwise distinct for $j=1, \ldots, 2 n-6$.

Lemma 2. Define

$$
\varphi:=\left(\frac{F^{\prime}}{F-1}-\frac{F^{\prime}}{F}\right)-\left(\frac{G^{\prime}}{G-1}-\frac{G^{\prime}}{G}\right),
$$

where $F:=R(f)$ and $G:=R(g)$, and $R(\omega)$ is given by (1.4). Suppose $n \geq 8$ if $f$ and $g$ are meromorphic, and $n \geq 6$ if $f$ and $g$ are entire. Then $\varphi \equiv 0$ implies $F \equiv G$.

Proof. Since we assume $\varphi \equiv 0$, integrating (2.3) yields

$$
\frac{1}{F}-\frac{A}{G} \equiv 1-A
$$

where $A \neq 0$ is a constant. As $F=R(f)$ and $G:=R(g)$, we know that $T(r, F)=n T(r, f)+O(1)$ and $T(r, G)=n T(r, g)+O(1)$, which together with (2.4) yields

$$
T(r, f)=T(r, g)+O(1) .
$$

Define $h_{1}:=1 / F$ and $h_{2}:=-A / G$. So, $h_{1}+h_{2} \equiv 1-A$. If $A \neq 1$, applying the second main theorem to $h_{1}$, plus (2.5), yields

$$
\begin{aligned}
T\left(r, h_{1}\right) \leq & \bar{N}\left(r, h_{1}\right)+\bar{N}\left(r, \frac{1}{h_{1}}\right)+\bar{N}\left(r, \frac{1}{h_{1}-(1-A)}\right)+S(r, f) \\
\leq & \bar{N}(r, F)+\bar{N}(r, G)+\bar{N}\left(r, \frac{1}{F}\right)+S(r, f) \\
\leq & \bar{N}(r, f)+\bar{N}\left(r, \frac{1}{f-\alpha}\right)+\bar{N}\left(r, \frac{1}{f-\beta}\right)+\bar{N}\left(r, \frac{1}{f}\right)+\bar{N}(r, g) \\
& +\bar{N}\left(r, \frac{1}{g-\alpha}\right)+\bar{N}\left(r, \frac{1}{g-\beta}\right)+S(r, f) \leq 7 T(r, f)+S(r, f),
\end{aligned}
$$

which implies that $n T(r, f) \leq 7 T(r, f)+S(r, f)$, a contradiction to our assumption that $n \geq 8$ if $f$ and $g$ are meromorphic.

If $f$ and $g$ are entire, then $\bar{N}(r, f)=O(1)$ and $\bar{N}(r, g)=O(1)$. A contradiction follows immediately, since now $n T(r, f) \leq 5 T(r, f)+S(r, f)$ but $n \geq 6$.

Lemma 3. Let $\varphi, F$ and $G$ be as in Lemma 2. If $\varphi \not \equiv 0$, and if two nonconstant meromorphic functions $f$ and $g$ satisfy $E_{k)}(\mathbf{S}, f)=E_{k)}(\mathbf{S}, g)$ for a positive integer $k$ and $\bar{E}(\infty, f)=\bar{E}(\infty, g)$, then

$$
N(r, f) \leq \frac{k+1}{k n-3 k-2}(T(r, f)+T(r, g))+S(r, f)+S(r, g) .
$$


Proof. Since $E_{k)}(\mathbf{S}, f)=E_{k)}(\mathbf{S}, g)$ for a positive integer $k$, we have $E_{k)}(1, F)=E_{k)}(1, G)$.

It is not difficult to show that

$$
N\left(r, 1 / h^{\prime}\right) \leq \bar{N}(r, h)+N(r, 1 / h)+S(r, h),
$$

where $h$ is a nonconstant meromorphic function.

In fact, from the lemma of logarithmic derivative, we have

$$
m(r, 1 / h) \leq m\left(r, 1 / h^{\prime}\right)+m\left(r, h^{\prime} / h\right) \leq m\left(r, 1 / h^{\prime}\right)+S(r, h) .
$$

Combining the above inequality with the first main theorem yields

$$
\begin{aligned}
N\left(r, 1 / h^{\prime}\right) & =T\left(r, h^{\prime}\right)-m\left(r, 1 / h^{\prime}\right)+O(1) \\
& \leq m\left(r, h^{\prime}\right)+N\left(r, h^{\prime}\right)-m(r, 1 / h)+S(r, h) \\
& \leq m(r, h)+N(r, h)+\bar{N}(r, h)+m\left(r, h^{\prime} / h\right)-m(r, 1 / h)+S(r, h) \\
& \leq T(r, h)+\bar{N}(r, h)-m(r, 1 / h)+S(r, h) \\
& \leq N(r, 1 / h)+\bar{N}(r, h)+S(r, h),
\end{aligned}
$$

which implies $(2.7)$.

Obviously, $m(r, \varphi)=S(r, f)+S(r, g)$. Rewrite $\varphi$ as

$$
\varphi=\frac{F^{\prime}}{F(F-1)}-\frac{G^{\prime}}{G(G-1)}
$$

From $(2.8)$ and the assumption $E_{k)}(1, F)=E_{k}(1, G)$, it is easy to see that all the poles of $\varphi$ are simple, and derive from the zeros of $F$ or $G$ and the sets $E_{(k+1}(1, F)$ and $E_{(k+1}(1, G)$, respectively, that is,

$$
\begin{aligned}
\leq & \bar{N}\left(r, \frac{1}{F}\right)+\bar{N}_{(k+1}\left(r, \frac{1}{F-1}\right)+\bar{N}\left(r, \frac{1}{G}\right) \\
& +\bar{N}_{(k+1}\left(r, \frac{1}{G-1}\right)+S(r, f)+S(r, g) \\
\leq & \bar{N}\left(r, \frac{1}{f}\right)+\sum_{j=1}^{n} \bar{N}_{(k+1}\left(r, \frac{1}{f-\omega_{j}}\right)+\bar{N}\left(r, \frac{1}{g}\right) \\
& +\sum_{j=1}^{n} \bar{N}_{(k+1}\left(r, \frac{1}{g-\omega_{j}}\right)+S(r, f)+S(r, g) \\
\leq & \bar{N}\left(r, \frac{1}{f}\right)+\bar{N}_{(k}\left(r, \frac{1}{f^{\prime}}\right)+\bar{N}\left(r, \frac{1}{g}\right)+\bar{N}_{(k}\left(r, \frac{1}{g^{\prime}}\right)+S(r, f)+S(r, g) \\
\leq & \bar{N}\left(r, \frac{1}{f}\right)+\frac{1}{k} N\left(r, \frac{1}{f^{\prime}}\right)+\bar{N}\left(r, \frac{1}{g}\right)+\frac{1}{k} N\left(r, \frac{1}{g^{\prime}}\right)+S(r, f)+S(r, g),
\end{aligned}
$$


where $\omega_{j}$ for $j=1, \ldots, n$ are the pairwise distinct roots of $P(\omega)$ given by (1.3).

Combining (2.7), (2.9) with the condition $\bar{E}(\infty, f)=\bar{E}(\infty, g)$ yields

$$
N(r, \varphi) \leq \frac{k+1}{k}(T(r, f)+T(r, g))+\frac{2}{k} \bar{N}(r, f)+S(r, f)+S(r, g) .
$$

Now, let $z_{0}$ be a pole of $f$ with multiplicity $p$, and hence a pole of $g$ with multiplicity $q$. Then $z_{0}$ is a pole of $F$ with multiplicity $(n-2) p$ and a pole of $G$ with multiplicity $(n-2) q$. By $(2.8), z_{0}$ is a zero of $\varphi$ with multiplicity at least $n-3$. Since we assume $\bar{E}(\infty, f)=\bar{E}(\infty, g)$, it follows that

$$
(n-3) \bar{N}(r, f) \leq N(r, 1 / \varphi) \leq T(r, \varphi)+O(1) .
$$

Hence, (2.10), (2.11) and $m(r, \varphi)=S(r, f)+S(r, g)$ yield $(2.6)$.

Lemma 4 (see [16]). Define

$$
\psi:=\left(\frac{F^{\prime \prime}}{F^{\prime}}-\frac{2 F^{\prime}}{F-1}\right)-\left(\frac{G^{\prime \prime}}{G^{\prime}}-\frac{2 G^{\prime}}{G-1}\right),
$$

where $F$ and $G$ are nonconstant meromorphic functions. If $\psi \equiv 0$, and

$$
\bar{N}(r, F)+\bar{N}(r, G)+\bar{N}\left(r, \frac{1}{F}\right) \leq \lambda T(r, F)+S(r, F)
$$

and

$$
\bar{N}(r, F)+\bar{N}\left(r, \frac{1}{G}\right)+\bar{N}\left(r, \frac{1}{F}\right) \leq \mu T(r, F)+S(r, F)
$$

for some real numbers $\lambda, \mu<1$, then either $F \equiv G$ or $F G \equiv 1$.

Proof. For convenience, we outline a proof. Since we assume $\psi \equiv 0$, integrating (2.12) yields

$$
\frac{1}{G-1}=\frac{A}{F-1}+B
$$

where $A(\neq 0)$ and $B$ are two constants. Rewrite it as

$$
G=\frac{(B+1) F+(A-B-1)}{B F+(A-B)} .
$$

We now distinguish the following three cases.

Case (i): $B \neq 0,-1$. If $A-B-1 \neq 0$, then

$$
\bar{N}\left(r, \frac{1}{F+\frac{A-B-1}{B+1}}\right)=\bar{N}\left(r, \frac{1}{G}\right)+O(1)
$$


by (2.16). Applying the second main theorem to $F$ yields

$$
\begin{aligned}
T(r, F) & \leq \bar{N}(r, F)+\bar{N}\left(r, \frac{1}{F}\right)+\bar{N}\left(r, \frac{1}{F+\frac{A-B-1}{B+1}}\right)+S(r, F) \\
& \leq \bar{N}(r, F)+\bar{N}\left(r, \frac{1}{G}\right)+\bar{N}\left(r, \frac{1}{F}\right) \leq \mu T(r, F)+S(r, F),
\end{aligned}
$$

a contradiction to (2.14) since $\mu<1$.

Hence, $A-B-1=0$. From $(2.16)$, we see that $\bar{N}(r, 1 /(F+1 / B))=$ $\bar{N}(r, G)+O(1)$. Similarly, we derive a contradiction to (2.13).

CASE (ii): $B=0$. If $A \neq 1$, we see that $\bar{N}(r, 1 /(F+A-1))=\bar{N}(r, 1 / G)$ $+O(1)$. Analogously, we derive a contradiction to $(2.14)$. So, $A=1$, and thus $F \equiv G$.

CASE (iii): $B=-1$. If $A \neq-1$, then $\bar{N}(r, 1 /(F-A-1))=\bar{N}(r, G)+$ $O(1)$. Analogously, a contradiction to (2.13) follows. Therefore, $A=-1$ and $F G \equiv 1$.

3. Proof of Theorem 3. Define $F:=R(f)$ and $G:=R(g)$, where $R(\omega)$ is given by (1.4). Since we assume $E_{3)}(\mathbf{S}, f)=E_{3)}(\mathbf{S}, g)$, it follows that $E_{3)}(1, F)=E_{3)}(1, G)$. Let $\psi$ be defined by $(2.12)$.

Firstly, we assume that $\psi \not \equiv 0$. Obviously, $E_{1}(1, F)=E_{1}(1, G)$ anyway. Let $z_{0}$ be a simple zero of $F-1$, and hence a simple zero of $G-1$. A routine calculation leads to $\psi\left(z_{0}\right)=0$. Therefore, noting that $m(r, \psi)=S(r, f)+$ $S(r, g)$ and that all the poles of $\psi$ are simple, we have

$$
\begin{aligned}
\bar{N}_{1)}\left(r, \frac{1}{F-1}\right) & =\bar{N}_{1)}\left(r, \frac{1}{G-1}\right) \\
& \leq N\left(r, \frac{1}{\psi}\right) \leq \bar{N}(r, \psi)+S(r, f)+S(r, g) .
\end{aligned}
$$

From the expression of $F$, we know that the poles of $F$ arise from the poles of $f$ and the zeros of $(f-\alpha)(f-\beta)$. Let $z_{\infty}$ be a simple pole of $F$. A routine calculation leads to

$$
\left.\left(\frac{F^{\prime \prime}}{F^{\prime}}-\frac{2 F^{\prime}}{F-1}\right)\right|_{z_{\infty}}=O(1) .
$$

Obviously, the simple zeros of $(f-\alpha)(f-\beta)$ are simple poles of $F$, while the multiple zeros of $(f-\alpha)(f-\beta)$ are zeros of $f^{\prime}$.

Differentiating $F$ and $G$ yields

$$
F^{\prime}=\frac{a(n-2) f^{n-1}(f-b)^{2} f^{\prime}}{n(n-1)(f-\alpha)^{2}(f-\beta)^{2}}, \quad G^{\prime}=\frac{a(n-2) g^{n-1}(g-b)^{2} g^{\prime}}{n(n-1)(g-\alpha)^{2}(g-\beta)^{2}} .
$$


Combining (2.12) and (3.2), together with the assumptions that $\psi \not \equiv 0$, $E_{3)}(1, F)=E_{3)}(1, G), \bar{E}(\infty, f)=\bar{E}(\infty, g)$ and similar discussions on $G$, yields

$$
\begin{aligned}
\bar{N}(r, \psi) \leq & \bar{N}(r, f)+\bar{N}_{(4}\left(r, \frac{1}{F-1}\right)+\bar{N}_{(4}\left(r, \frac{1}{G-1}\right) \\
& +\bar{N}\left(r, \frac{1}{f}\right)+\bar{N}\left(r, \frac{1}{f-b}\right)+\bar{N}\left(r, \frac{1}{g}\right)+\bar{N}\left(r, \frac{1}{g-b}\right) \\
& +N_{0}\left(r, \frac{1}{f^{\prime}}\right)+N_{0}\left(r, \frac{1}{g^{\prime}}\right)+S(r, f)+S(r, g)
\end{aligned}
$$

where $N_{0}\left(r, 1 / f^{\prime}\right)$ denotes the counting function of the zeros of $f^{\prime}$ that are not the zeros of $f(f-b)$ and $F-1$, and $N_{0}\left(r, 1 / g^{\prime}\right)$ denotes the counting function of the zeros of $g^{\prime}$ that are not the zeros of $g(g-b)$ and $G-1$.

Applying the second main theorem to $f$ and $g$ jointly with $n+3$ values $0, b, \infty$ and the zeros of $P(\omega)$ defined by (1.3) yields

$$
\begin{aligned}
&(n+1)(T(r, f)+T(r, g)) \\
& \leq \bar{N}(r, f)+\bar{N}\left(r, \frac{1}{F-1}\right)+\bar{N}\left(r, \frac{1}{f}\right)+\bar{N}\left(r, \frac{1}{f-b}\right) \\
&+\bar{N}(r, g)+\bar{N}\left(r, \frac{1}{G-1}\right)+\bar{N}\left(r, \frac{1}{g}\right)+\bar{N}\left(r, \frac{1}{g-b}\right) \\
&-N_{0}\left(r, \frac{1}{f^{\prime}}\right)-N_{0}\left(r, \frac{1}{g^{\prime}}\right)+S(r, f)+S(r, g) .
\end{aligned}
$$

From $(3.1),(3.3),(3.4)$ and $\bar{E}(\infty, f)=\bar{E}(\infty, g)$, we have

$$
\begin{aligned}
& (n+1)(T(r, f)+T(r, g)) \\
\leq & 3 \bar{N}(r, f)+2 \bar{N}\left(r, \frac{1}{f}\right)+2 \bar{N}\left(r, \frac{1}{f-b}\right)+2 \bar{N}\left(r, \frac{1}{g}\right) \\
+ & 2 \bar{N}\left(r, \frac{1}{g-b}\right)+\bar{N}\left(r, \frac{1}{F-1}\right)+\bar{N}\left(r, \frac{1}{G-1}\right)+\bar{N}_{(4}\left(r, \frac{1}{F-1}\right) \\
+ & \bar{N}_{(4}\left(r, \frac{1}{G-1}\right)-N_{1)}\left(r, \frac{1}{F-1}\right)+S(r, f)+S(r, g) .
\end{aligned}
$$

It is not difficult to see that

$$
\begin{aligned}
& \bar{N}\left(r, \frac{1}{F-1}\right)-\frac{1}{2} N_{1)}\left(r, \frac{1}{F-1}\right)+\bar{N}_{(4}\left(r, \frac{1}{F-1}\right) \leq \frac{1}{2} N\left(r, \frac{1}{F-1}\right), \\
& \bar{N}\left(r, \frac{1}{G-1}\right)-\frac{1}{2} N_{1)}\left(r, \frac{1}{G-1}\right)+\bar{N}_{(4}\left(r, \frac{1}{G-1}\right) \leq \frac{1}{2} N\left(r, \frac{1}{G-1}\right),
\end{aligned}
$$


which implies that

$$
\begin{aligned}
\bar{N}\left(r, \frac{1}{F-1}\right)+ & \bar{N}\left(r, \frac{1}{G-1}\right)+\bar{N}_{(4}\left(r, \frac{1}{F-1}\right)+\bar{N}_{(4}\left(r, \frac{1}{G-1}\right) \\
& -N_{1)}\left(r, \frac{1}{F-1}\right) \leq \frac{n}{2}(T(r, f)+T(r, g))+O(1) .
\end{aligned}
$$

Noting that

$$
\begin{aligned}
& 2 \bar{N}\left(r, \frac{1}{f}\right)+2 \bar{N}\left(r, \frac{1}{f-b}\right) \leq 4 T(r, f)+O(1), \\
& 2 \bar{N}\left(r, \frac{1}{g}\right)+2 \bar{N}\left(r, \frac{1}{g-b}\right) \leq 4 T(r, g)+O(1),
\end{aligned}
$$

we deduce from (3.5) and (3.6) that

$$
(n-6)(T(r, f)+T(r, g)) \leq 6 \bar{N}(r, f)+S(r, f)+S(r, g) .
$$

We have $\varphi \not \equiv 0$. Indeed, otherwise, from the conclusions of Lemma 2, we have $F \equiv G$, and hence $\psi \equiv 0$. From (2.6) and (3.7), and noting $k=3$, we conclude that

$$
\frac{3 n^{2}-29 n+42}{3 n-11}(T(r, f)+T(r, g)) \leq S(r, f)+S(r, g),
$$

a contradiction to the assumption $n \geq 8$.

Therefore, $\psi \equiv 0$. By (2.15), we know that $T(r, F)=T(r, G)+O(1)$. So,

$$
\begin{aligned}
\bar{N}(r, F)+\bar{N}(r, G)+\bar{N}\left(r, \frac{1}{F}\right) & \leq \frac{7}{n} T(r, F)+S(r, F), \\
\bar{N}(r, F)+\bar{N}\left(r, \frac{1}{G}\right)+\bar{N}\left(r, \frac{1}{F}\right) & \leq \frac{5}{n} T(r, F)+S(r, F) .
\end{aligned}
$$

By the conclusions of Lemma 4 , we have either $F \equiv G$ or $F G \equiv 1$.

We now distinguish the following two cases.

CASE (i): $F G \equiv 1$. It is obvious that

$$
\frac{f^{n}}{(f-\alpha)(f-\beta)} \frac{g^{n}}{(g-\alpha)(g-\beta)} \equiv \frac{n^{2}(n-1)^{2}}{a^{2}} .
$$

By (3.9) and noting $\bar{E}(\infty, f)=\bar{E}(\infty, g)$, we find that $\infty$ is a Picard value of $f$, and zeros of $(f-\alpha)(f-\beta)$ are of multiplicities at least $n$. Therefore, applying the second main theorem to $f$ yields

$$
\begin{aligned}
T(r, f) & \leq \bar{N}(r, f)+\bar{N}\left(r, \frac{1}{f-\alpha}\right)+\bar{N}\left(r, \frac{1}{f-\beta}\right)+S(r, f) \\
& \leq \frac{1}{n} N\left(r, \frac{1}{f-\alpha}\right)+\frac{1}{n} N\left(r, \frac{1}{f-\beta}\right)+S(r, f) \leq \frac{2}{n} T(r, f)+S(r, f),
\end{aligned}
$$

a contradiction to the assumption $n \geq 8$. 
CASE (ii): $F \equiv G$. It is obvious that

$$
\begin{aligned}
n(n-1) f^{2} g^{2}\left(f^{n-2}-g^{n-2}\right)-2 b n( & -2) f g\left(f^{n-1}-g^{n-1}\right) \\
& +b^{2}(n-1)(n-2)\left(f^{n}-g^{n}\right) \equiv 0 .
\end{aligned}
$$

Set $h=f / g$. Then substituting $f=h g$ into the above equation yields

$$
\begin{aligned}
& n(n-1) h^{2}\left(h^{n-2}-1\right) g^{2}-2 b n(n-2) h\left(h^{n-1}-1\right) g \\
& +b^{2}(n-1)(n-2)\left(h^{n}-1\right) \equiv 0 .
\end{aligned}
$$

If $h$ is not a constant, then from (3.10) we have

$$
\left\{n(n-1) h\left(h^{n-2}-1\right) g-b n(n-2)\left(h^{n-1}-1\right)\right\}^{2} \equiv-b^{2} n(n-2) Q(h),
$$

where $Q(\omega)$ is given by (2.1). Applying the conclusions of Lemma 1 to (3.11) yields

$$
\begin{aligned}
& \left\{n(n-1) h\left(h^{n-2}-1\right) g-b n(n-2)\left(h^{n-1}-1\right)\right\}^{2} \\
& \quad \equiv-b^{2} n(n-2)(n-1)^{2}(h-1)^{4}\left(h-\gamma_{1}\right)\left(h-\gamma_{2}\right) \cdots\left(h-\gamma_{2 n-6}\right),
\end{aligned}
$$

where $\gamma_{j} \in \mathbb{C} \backslash\{0,1\}$ are pairwise distinct and $Q\left(\gamma_{j}\right)=0$ for $j=1, \ldots, 2 n-6$.

It is easily seen from (3.12) that zeros of $h-\gamma_{j}(j=1, \ldots, 2 n-6)$ are of multiplicities at least 2 . Therefore, applying the second main theorem to $h$ yields

$$
\begin{aligned}
(2 n-8) T(r, h) & \leq \sum_{j=1}^{2 n-6} \bar{N}\left(r, \frac{1}{h-\gamma_{j}}\right)+S(r, h) \\
& \leq \frac{1}{2} \sum_{j=1}^{2 n-6} N\left(r, \frac{1}{h-\gamma_{j}}\right)+S(r, h) \\
& \leq(n-3) T(r, h)+S(r, h),
\end{aligned}
$$

a contradiction to the assumption $n \geq 8$.

Hence, $h$ is a constant. From (3.10), we see that $h^{n-2}-1=0, h^{n-1}-1=0$ and $h^{n}-1=0$ simultaneously, which means $h=1$. So, $f \equiv g$.

4. Proof of Theorem 4. Similar to the proof Theorem 3, we have

$$
\begin{aligned}
& (n+1)(T(r, f)+T(r, g)) \\
\leq & 3 \bar{N}(r, f)+2 \bar{N}\left(r, \frac{1}{f}\right)+2 \bar{N}\left(r, \frac{1}{f-b}\right)+2 \bar{N}\left(r, \frac{1}{g}\right) \\
+ & 2 \bar{N}\left(r, \frac{1}{g-b}\right)+\bar{N}\left(r, \frac{1}{F-1}\right)+\bar{N}\left(r, \frac{1}{G-1}\right)+\bar{N}_{(3}\left(r, \frac{1}{F-1}\right) \\
+ & \bar{N}_{(3}\left(r, \frac{1}{G-1}\right)-N_{1)}\left(r, \frac{1}{F-1}\right)+S(r, f)+S(r, g) .
\end{aligned}
$$


It is not difficult to see that

$$
\begin{array}{r}
\bar{N}\left(r, \frac{1}{F-1}\right)-\frac{1}{2} N_{1)}\left(r, \frac{1}{F-1}\right)+\frac{1}{2} \bar{N}_{(3}\left(r, \frac{1}{F-1}\right) \\
\leq \frac{1}{2} N\left(r, \frac{1}{F-1}\right) \\
\bar{N}\left(r, \frac{1}{G-1}\right)-\frac{1}{2} N_{1)}\left(r, \frac{1}{G-1}\right)+\frac{1}{2} \bar{N}_{(3}\left(r, \frac{1}{G-1}\right) \\
\leq \frac{1}{2} N\left(r, \frac{1}{G-1}\right) .
\end{array}
$$

From (2.7), we have

$$
\begin{aligned}
\bar{N}_{(3}\left(r, \frac{1}{F-1}\right) & \leq \sum_{j=1}^{n} \bar{N}_{(3}\left(r, \frac{1}{f-\omega_{j}}\right) \leq \frac{1}{2} N\left(r, \frac{1}{f^{\prime}}\right) \\
& \leq \frac{1}{2} \bar{N}(r, f)+\frac{1}{2} N\left(r, \frac{1}{f}\right)+S(r, f) \\
& \leq \frac{1}{2} \bar{N}(r, f)+\frac{1}{2} T(r, f)+S(r, f),
\end{aligned}
$$

where $\omega_{j}$ for $j=1, \ldots, n$ are the pairwise distinct roots of $P(\omega)$ given by (1.3).

Similarly, we have

$$
\bar{N}_{(3}\left(r, \frac{1}{G-1}\right) \leq \frac{1}{2} \bar{N}(r, g)+\frac{1}{2} T(r, g)+S(r, g) .
$$

Combining (4.2)-(4.5) with the assumptions $E_{2)}(1, F)=E_{2}(1, G)$ and $\bar{E}(\infty, f)=\bar{E}(\infty, g)$ yields

$$
\begin{aligned}
\bar{N}\left(r, \frac{1}{F-1}\right)+\bar{N}\left(r, \frac{1}{G-1}\right) & +\bar{N}_{(3}\left(r, \frac{1}{F-1}\right) \\
& +\bar{N}_{(3}\left(r, \frac{1}{G-1}\right)-N_{1)}\left(r, \frac{1}{F-1}\right) \\
\leq & \frac{1}{2} \bar{N}(r, f)+\frac{2 n+1}{4}(T(r, f)+T(r, g))+O(1) .
\end{aligned}
$$

Substituting the above inequality into (4.1) yields

$$
(2 n-13)(T(r, f)+T(r, g)) \leq 14 \bar{N}(r, f)+S(r, f)+S(r, g) .
$$

Applying the conclusions of Lemma 2 to (4.6) with $k=2$ yields

$$
\frac{2 n^{2}-21 n+31}{n-4}(T(r, f)+T(r, g)) \leq S(r, f)+S(r, g),
$$

a contradiction to the assumption $n \geq 9$. 
5. Proof of Theorem 5. Analogously to the proof Theorem 3, we have (5.1)

$$
\begin{aligned}
& (n+1)(T(r, f)+T(r, g)) \\
\leq & 3 \bar{N}(r, f)+2 \bar{N}\left(r, \frac{1}{f}\right)+2 \bar{N}\left(r, \frac{1}{f-b}\right)+2 \bar{N}\left(r, \frac{1}{g}\right) \\
+ & 2 \bar{N}\left(r, \frac{1}{g-b}\right)+\bar{N}\left(r, \frac{1}{F-1}\right)+\bar{N}\left(r, \frac{1}{G-1}\right)+\bar{N}_{(2}\left(r, \frac{1}{F-1}\right) \\
+ & \bar{N}_{(2}\left(r, \frac{1}{G-1}\right)-N_{1)}\left(r, \frac{1}{F-1}\right)+S(r, f)+S(r, g) .
\end{aligned}
$$

It is not difficult to see that

$$
\begin{aligned}
& \bar{N}\left(r, \frac{1}{F-1}\right)-\frac{1}{2} N_{1)}\left(r, \frac{1}{F-1}\right) \leq \frac{1}{2} N\left(r, \frac{1}{F-1}\right), \\
& \bar{N}\left(r, \frac{1}{G-1}\right)-\frac{1}{2} N_{1)}\left(r, \frac{1}{G-1}\right) \leq \frac{1}{2} N\left(r, \frac{1}{G-1}\right) .
\end{aligned}
$$

From (2.7) and (4.4), we have

$$
\begin{aligned}
\bar{N}_{(2}\left(r, \frac{1}{F-1}\right) & \leq \sum_{j=1}^{n} \bar{N}_{(2}\left(r, \frac{1}{f-\omega_{j}}\right) \leq N\left(r, \frac{1}{f^{\prime}}\right) \\
& \leq \bar{N}(r, f)+T(r, f)+S(r, f),
\end{aligned}
$$

where $\omega_{j}$ are as before for $j=1, \ldots, n$.

Analogously, we have

$$
\bar{N}_{(2}\left(r, \frac{1}{G-1}\right) \leq \bar{N}(r, g)+T(r, g)+S(r, g) .
$$

Combining (5.2)-(5.5) with the assumptions $E_{1)}(1, F)=E_{1)}(1, G)$ and $\bar{E}(\infty, f)=\bar{E}(\infty, g)$ yields

$$
\begin{aligned}
\bar{N}\left(r, \frac{1}{F-1}\right)+\bar{N}\left(r, \frac{1}{G-1}\right)+ & \bar{N}_{(2}\left(r, \frac{1}{F-1}\right) \\
& +\bar{N}_{(2}\left(r, \frac{1}{G-1}\right)-N_{1)}\left(r, \frac{1}{F-1}\right) \\
\leq 2 \bar{N}(r, f) & +\frac{n+2}{2}(T(r, f)+T(r, g))+O(1) .
\end{aligned}
$$

Substituting the above inequality into $(5.1)$ yields

$$
(n-8)(T(r, f)+T(r, g)) \leq 10 \bar{N}(r, f)+S(r, f)+S(r, g) .
$$

Applying the conclusions of Lemma 2 to (5.6) with $k=1$ yields

$$
\frac{n^{2}-13 n+20}{n-5}(T(r, f)+T(r, g)) \leq S(r, f)+S(r, g),
$$

a contradiction to the assumption $n \geq 12$. 
6. Proof of Theorems 1 and 2. Analogously to the proof of Theorem 4 , we have

$$
(2 n-13)(T(r, f)+T(r, g)) \leq S(r, f)+S(r, g),
$$

since the terms $\bar{N}(r, f)$ and $\bar{N}(r, g)$ are $O(1)$. Clearly, this contradicts the assumption that $n \geq 7$ and terminates the proof of Theorem 1 .

For the proof of Theorem 2, by the proofs of Theorems 1 and 5, we see that

$$
(n-8)(T(r, f)+T(r, g)) \leq S(r, f)+S(r, g),
$$

which immediately yields a contradiction to $n \geq 9$.

Acknowledgements. The first author would like to express his genuine gratitude to his parents for their love and financial support.

\section{References}

[1] M. L. Fang and H. Gou, On meromorphic functions sharing two values, Analysis (Munich) 17 (1997), 355-366.

[2] G. Frank and M. Reinders, A unique range set for meromorphic functions with 11 elements, Complex Variables Theory Appl. 37 (1998), 185-193.

[3] F. Gross, On the distribution of values of meromorphic functions, Trans. Amer. Math. Soc. 131 (1968), 199-214.

[4] - Factorization of Meromorphic Functions and Some Open Problems, Springer, Berlin, 1977.

[5] F. Gross and C. C. Yang, On preimage and range sets of meromorphic functions, Proc. Japan Acad. (Ser. A) 58 (1982), 17-20.

[6] W. K. Hayman, Meromorphic Functions, Clarendon Press, Oxford, 1964.

[7] P. Li and C. C. Yang, On the unique range sets for meromorphic functions, Proc. Amer. Math. Soc. 124 (1996), 177-185.

[8] —, - Some further results on the unique range sets for meromorphic functions, Kodai Math. J. 18 (1995), 437-450.

[9] E. Mues and M. Reinders, Meromorphic functions sharing one value and unique range sets, ibid. 18 (1995), 515-522.

[10] C. C. Yang and H. X. Yi, Uniqueness Theory of Meromorphic Functions, Science Press \& Kluwer, Beijing and Dordrecht, 2003.

[11] H. X. Yi, Uniqueness of meromorphic functions and a question of Gross, Sci. China (Ser. A) 37 (1994), 802-813.

[12] -, On a question of Gross, Sci. China (Ser. A) 38 (1995), 8-16.

[13] - Unicity theorems for meromorphic functions or entire functions II, Bull. Austral. Math. Soc. 52 (1995), 215-224.

[14] - , The unique range sets of entire or meromorphic functions, Complex Variables Theory Appl. 28 (1995), 13-21.

[15] - On a question of Gross concerning uniqueness of entire functions, Bull. Austral. Math. Soc. 57 (1998), 343-349. 
[16] H. X. Yi, Meromorphic functions that share two sets, Acta Math. Sinica 45 (2002), $75-82$.

School of Mathematics and System Sciences

Shandong University

Jinan 250100, Shandong, People's Republic of China

E-mail: k.l.han@tom.com

hxyi@sdu.edu.cn

Received 9.3.2007

and in final form 7.12.2007 